\title{
Flow quantification
}

\section{J.M. Rubin}

Department of Radiology, University of Michigan, 1500E Medical Centre DR, Ann Arbor, MI 48109, USA

\section{Introduction}

Flow measurements have been possible in ultrasound ever since the advent of Doppler; however, the measurements have been problematic and error prone. This has lead to compromises in the flow measurements that have generally been performed. These compromises have included flow velocity measurements, which are a direct outgrowth of Doppler, rather than true volume flow, and semi-quantitative parameters such as resistive indices (RIs) and pulsatility indices (PIs). Flow velocities have direct value in that conservation of volume flow due to autoregulating areas produces increases in local flow velocities at areas of stenosis. Such changes have proven very useful for the diagnosis. Also because of the Bernoulli equation, peak flow velocities can be used to estimate pressure drops in the correct circumstances such at the aortic and pulmonary valves. Although attractive because they are simple to perform, RIs and PIs have very frequently provided less than totally useful information. This may be because these measurements are only empirical, and really do not measure what they are claim to, i.e. vascular resistance. Ultimately, one would like to measure flow directly, and recent developments have moved closer to this goal.

\section{Measuring flow volume}

The first volume flow estimates were made with Doppler. The ubiquitous Doppler equation is:

$\Delta \mathrm{f}=2(\mathrm{v} / \mathrm{c}) \mathrm{f}_{\mathrm{o}} \cos \Theta$

where $\Delta \mathrm{f}$ is the Doppler shifted frequency, $\mathrm{v}$ is the velocity of the blood flow, $c$ is the speed of sound, $\mathrm{f}_{\mathrm{o}}$ is the carrier frequency of the Doppler and $\Theta$ is the Doppler angle. Solving for velocity and opening the range gate to include the entire width of a blood vessel, one can estimate the mean velocity through the range gate. By assuming the vessel is circular in cross section, one can estimate the radius of the blood vessel, calculate the cross-sectional area of the vessel, and by multiplying flow determine the mean velocity through the cross section by the area of the cross section. Although true in theory, these estimates lead to many problems in practice. Firstly, the cross section is almost never circular making the area estimate imprecise, and secondly, the velocity profile is hardly ever cylindrically symmetric, making the velocity sampling assumptions wrong. The usual machine-programmed volume estimating routines assume a circular cross section. Even if this assumption is correct, the effect of the error multiplies with small radii. The fractional error is $\Delta \mathrm{A} / \mathrm{A}=2 \Delta \mathrm{r} / \mathrm{r}$, where $\mathrm{A}$ is area and $\mathrm{r}$ is radius. Thus, for example, if a vessel has a radius of $5 \mathrm{~mm}$ and the error in measuring the radius is $\pm 1 \mathrm{~mm}$, then the fractional error will be $40 \%$. Such problems alone have discouraged use of this method. An additional problem is estimating the Doppler angle, yet, certain angle-independent flow techniques can theoretically decrease this problem. However, major issues still remain.

A novel solution to this problem had been proposed some years ago by Hottinger and Meindl [1]. They proposed using two different beam profiles to solve the geometry problem. They used a beam with large cross section, i.e. larger than the cross section of the vessel itself, to uniformly sample across the entire vessel. They could then measure the entire Doppler power in this cross section and could also measure a mean Doppler shift that included all of the velocity components in the cross section. They then used a very small, highly focused beam to sample the Doppler power from a site that was totally within the vessel. They then used this power to calculate the true vessel cross section, independent of geometry. They could, hence, obtain a true volume flow measurement. The method worked in flow tubes but has not been employed clinically. The big problems relate to accurate estimation and control of the two beam profiles, and the underlying assumption that the Doppler power in the small sample volume would reflect the true power from $100 \%$ blood across the blood vessel. Unfortunate- 
ly, fluctuations in Doppler power are not only due to the fractions of any given location that contain blood, which is what this method requires. An equally important, if not more important, contributor to these variations is rouleaux (see below). Due to red blood cell clumping, the variations in Doppler power are a function of the degree of clumping which does not reflect the desired geometry parameter one wishes to measure $[2,3,4,5,6]$. However, a method similar to the Hottinger and Meidel [1] technique may now be useful and possible using contrast agents.

\section{Other methods for volume flow measurements}

Other velocity-estimating methods which employ speckle tracking have also been employed, and some have reached clinical applications. Speckle is due to the interference of the backscattering patterns of subwave scatterers. The interference pattern changes as scatterers move in and out of sampling sites in the beam. These patterns tend to maintain their shapes for certain distance ranges, and they can be tracked as a function of time to estimate local velocity. This is a broadband technique rather than the more standard narrow-band Doppler methods. This permits grayscale-type velocity mappings giving high spatial resolution with high frame rates. A one-dimensional technique tracks motion along lines of sight emanating from a scanhead similar to standard color or spectral Doppler [7]. In many ways it has similar limitations to Doppler including angle dependence. In 1D this method is now used clinically, and it can be extended to 2D with more sophisticated tracking methods $[8,9]$. Volume flow estimates can be obtained with both methods. In the clinically implemented 1D measure, a velocity profile is drawn, and the mean velocity calculated from that profile. The method still relies on many of the same assumptions as standard spectral Doppler, and although the in-plane spatial and temporal resolutions are bound to be better, out-of-plane flow contributions suffer from the same assumptions as standard duplex Doppler estimates of volume flow.

True volume flow is defined as the total flux across a surface intersecting the vessel. The flux is based on the component of the velocity normal to the intersecting plane. This is interesting in that the measurement is angle independent because of the following:

$$
\text { Volume flow }=\oiint \mathrm{v} \bullet n d A
$$

where $\mathrm{v}$ is the same as in Eq. (1), $\mathrm{dA}$ a small area in the surface with unit normal $\mathrm{n}$, and . is the dot product. If the Doppler frequency, $\Delta \mathrm{f}$, at any position, is multiplied by the tipped infinitesimal area, dA' where $\mathrm{dA}^{\prime}=\mathrm{dA} /$ $\cos \Theta$, the result is:

$$
2(\mathrm{v} / \mathrm{c}) \mathrm{f}_{\mathrm{o}} \cos \Theta(\mathrm{dA} / \cos \Theta)
$$

The cosine terms cancel leaving the component of volume flow times a constant which is $2 \mathrm{f}_{\mathrm{o}} / \mathrm{c}$. Hence, volume flow is measured independent of angle. The problem is that the component of the flow velocity required here is exactly normal to the standard Doppler angle, which lies within the measurement plane, not orthogonal to it as is required by this method. The capacity to obtain this orthogonal velocity component, especially in realtime, is not simple and may require a true 3D acquisition to do it as is now under development $[10,11]$.

\section{Perfusion imaging}

Perfusion imaging has long been considered the "holy grail" for ultrasound flow detection. The standard measure of perfusion is in units of millilitres of fluid per second/100 g of tissue. Typically, the 100 grams of tissue is converted into volume using densities of the order of water, i.e. $1 \mathrm{~g} / \mathrm{ml}$; hence, the overall units are measured per second. A simple way of thinking about this is as a fractional blood volume in a region divided by the mean transit time through the region where the region is scaled to $100 \mathrm{ml}$ of tissue. The fractional blood volume is the fraction of the tissue of interest that is composed of blood. A measure of fractional blood volume can be made using contrast agents and comparing the amount of contrast agent in the tissue to a blood standard. Such techniques have been employed in nuclear medicine, MRI and CT $[12,13,14]$. A general problem with the method is that the indicator or contrast agent must remain intravascular for the method to be valid. If the indicator diffuses into the tissue, the method will provide an incorrect answer. Secondly, the transit time through tissue is strongly modified by spreading of the bolus of contrast agent, and the time dependence of the bolus of injection. This causes an underestimate of the mean transit time.

\section{Measuring fractional blood volume}

Fractional blood volume estimates can be made with ultrasound $[15,16]$. The advantages of ultrasonography are that the imaging does not require contrast agents and the red blood cells, which act as the ultrasound contrast agents, are intravascular. It is then possible to make this measurement by identifying a region of interest, and then scanning that region using power Doppler. Then by locating a large blood vessel within or near the region of interest, the mean power in the region of interest can be normalized by the mean power in the blood vessel to estimate the fractional blood volume in the tissue. Another advantage of this method is that the normalization compensates for things such as overlying attenuation and transducer aperture effects, all of which can confound standard ultrasound flow quantification strategies.

Although functional in theory, this method does have some problems. The first problem is that power Doppler depends on the presence of measurable Doppler shifts in order to make the estimate. Velocities from which Doppler shifts are too low to measure will not contribute to the power measurement. Capillary flow velocity 
is of the order of $1 \mathrm{~mm} / \mathrm{s}$, which will not typically generate a measureable Doppler shift, and hence will not be seen with this method. In addition, the estimate is highly dependent on the normalization value that is chosen, which is even more problematic than at first review because the $100 \%$ blood value varies across a blood vessel. This is because of rouleaux $[2,3,4,5]$. Rouleaux is the process of clumping of flowing red blood cells, and it is highly dependent on the shear rate across a blood vessel. Since there is more shear near the borders of vessels than in the centre, the Doppler power is higher in the centre of a flow stream than near the edges independent of things such as partial-volume averaging. Thus, the issue of which value to take to use for normalizing becomes a problem $[15,16]$.

This presumably can be overcome by using contrast agents. Ultrasound contrast agents are totally intravascular. They are spherical gas bubbles so they do not clump like biconcave discs, the red blood cells, and if they radiate ultrasound non-linearly, they can produce signals without moving; hence, capillary flow may be detectable and the normalization should be robust. It may be necessary to accomplish these measurements without breaking bubbles, which may create problems in themselves, although some measurements rely on bubble destruction. Many of these measurements can be done in grayscale, greatly increasing the dynamic range of the detectable signals, making the measurements more accurate.

\section{Measuring mean transit time}

The other major component of perfusion is an estimate of the mean transit time through the tissue. Estimates of this quantity have been attempted using almost all imaging modalities. The typical method is to try to measure the transit time passage after a bolus injection of contrast agent. Without making assumptions about distribution of the agent using multicompartmental models, the best estimates are generally those that incorporate a contrast agent that is totally intravascular, i.e. does not diffuse into tissue. This simplifies the transit time estimate, and is an advantage for bubble-based ultrasound contrast agents which are totally intravascular. Generally, the issue then becomes one of separating out the effects of the tissue transit time from those of the contrast injection. The bolus transit measurement is a composite of these and is generally defined in linear theory as the concentration of material in the tissue given by the convolution of the bolus concentration distribution and the tissue response impulse function $[17,18]$. Extracting the impulse response function, which leads directly to the mean transit time, from the shape of the bolus mathematically requires deconvolution of the measured intensity of the backscattered ultrasound energy. This is problematic, since it often leads to division by zero; hence, most people who attempt this measure make assumptions about the injection distribution or tissue transit properties.

\section{New imaging techniques}

Recently, new bubble destruction techniques have been developed that permit the generation of very sharp boluses using ultrasound contrast agents. Equally, continuous infusions of contrast agent can be modified to produce positive or negative boluses by selective destruction of the contrast agent; hence, it is possible to get mean transit time estimates without making assumptions about the shape of the bolus [19].

Some new methods that take advantage of the fact that ultrasound contrast agents are composed of compressible bubbles which can be rapidly destroyed or made to respond non-linearly when insonified to yield differential signals during insonification are flash-echo techniques, loss of correlation (LOC), and pulse inversion imaging. In flash-echo methods, bubbles are imaged with high-amplitude pulses after time delays. The initial imaging pulse sees a large number density of bubbles at a given position, producing a large backscattered amplitude. This pulse, however, destroys many or all of these bubbles, so that subsequent closely spaced pulses see far fewer bubbles and hence have a weaker backscattered signal. If the pulses are turned off or the pulse amplitude is turned down below the bubble destruction level for a period of time, then the backscattered intensity from a repeated strong pulse will be an estimate of the wash-in and wash-out dynamics of the tissue. By varying the hard pulse interval, these dynamics can be measured. Loss of correlation imaging uses bubble destruction as its signal source but is not intended to provide quantitative flow information [20].

Pulse inversion methods also rely on the non-linear response of bubbles to an external sound field. With this method, a broadband pulse is transmitted and its reflection recorded. A short time later, a second pulse with a $180^{\circ}$ phase inversion is transmitted. These two pulses are added and an image formed based on the result, where non-moving soft tissue will cancel [21]. In fact, if this is regarded as if it were Doppler, non-moving tissue appears to have aliased because of the $\pi$ phase shift between pulses; hence, a filter that removes aliased signals will further improve clutter rejection. Anything that moves will not subtract, and since bubbles respond non-linearly, cancellation does not occur. A manifestation of the non-linearity is that even harmonics will also not cancel. This method has produced dramatic images of the blood flow, particularly in the myocardium.

A further addition of this method employs Doppler processing that cancels zero frequency shift in the Doppler [22]. This cancels the tissue harmonic as well, leaving only the bubbles due to their highly non-linear response. This improves the clutter rejection even further.

Another flow quantification technique which may have value with the use of contrast agents is speckle decorrelation imaging. This is not a new technique. It was originally described in 1974 by Atkinson and Berry [23], and there have been earlier attempts to employ it in clinical ultrasound imaging by Bamber et al. [24]. The method works based on the fluctuations in backscatter intensity of the speckle within a given position 
due to motion at that position. This fluctuation is a measure of this motion, and this motion can be quantified by performing frame-to-frame or pulse-to-pulse correlation measurements. It should be noted that this method is fundamentally different from the well-known speckle-tracking techniques described earlier $[7,8,9]$.

The motion can be due to material flowing or moving at the measurement site or conversely due to the motion of the ultrasound transducer itself over the target. The latter implementation has been used to estimate the rate of translation of a scanhead in the out-of-plane direction for 3D imaging [25]. The greatest advantages of the technique are that it could potentially measure very slow flows, on the order of capillary flow rates, it is inherently three dimensional and it appears to be less angle dependent than Doppler measurements.

The biggest problem with this method for blood flow is clutter rejection. Although it will theoretically work with the signal from only red blood cells, the backscatter is too small to detect this signal above the stationary background in any but the largest vessels with significant rouleaux such as the inferior vena cava. These problems can be overcome with contrast agents. The other major problem is that anything that changes the bubble/scatterer distribution in the tissue will cause the signal to decorrelate. This can be caused by movement of the bubbles in the flow stream due to external sources such as the radiation force from the transducer. This effect is probably small, especially in the small blood vessels where this method would be employed. The bigger problem is bubble breakage. This definitely affects the speckle distribution, and decorrelates the signal. To make this method work optimally, very stable contrast bubbles need to be used.

\section{References}

1. Hottinger CF, Meindel J (1979) Blood flow measurement using the attenuation-compensated volume flowmeter. Ultrasonic Imaging 1: 1-15

2. Cloutier G, Qin Z (1997) Ultrasound backscattering from nonaggregating and aggregating erythrocytes: a review. Biorheology $34: 443-470$

3. Sigel B, Machi J, Beitler J, Justin J (1983) Red cell aggregation as a cause of blood-flow echogenicity. Radiology 148: 799-802

4. Shung K, Cloutier G, Lim C (1992) The effects of hematocrit, shear rate, and turbulence on ultrasound Doppler spectrum from blood. IEEE Trans Biomed Eng 39: 462-469

5. Shung K (1993) In vitro experiment results on ultrasonic scattering in biological tissues. In: Shung K, Thieme G (eds) Ultrasonic scattering in biological tissues. CRC Press, Boca Raton, pp 291-312

6. Shung K, Kuo I, Cloutier G (1993) Ultrasound scattering properties of blood. In: Roelandt J, Gussenhoven E, Born N (eds) Intravascular ultrasound. Kluwer, Dordrecht, pp 119-139
7. Bonnefous O, Pesque P (1986) Time domain formulation of pulse-Doppler ultrasound and velocity estimation by cross-correlation. Ultrasonic Imaging 8: 73-85

8. Bohs LN, Trahey GE (1991) A novel method for angle independent ultrasonic imaging of blood flow and tissue motion. IEEE Trans Biomed Eng 38: 280-286

9. Bohs LN, Friemel BH, McDermott BA, Trahey GE (1993) A real time system for quantifying and displaying two-dimensional velocities using ultrasound. Ultrasound Med Biol 19: 751-761

10. Smith SW, Trahey GE, Ramm OT von (1992) Two-dimensional arrays for medical ultrasound. Ultrasonic Imaging 14: 213-233

11. Von Ramm OT, Smith SW (1990) Real time volumetric ultrasound imaging system. J Digit Imaging 3: 261-266

12. Miles KA, Hayball MP, Dixon AK (1993) Functional images of hepatic perfusion obtained with dynamic CT. Radiology 188: 405-411

13. Ishii Y, MacIntyre WJ (1971) Analytical approach to dynamic radioisotope recording. J Nucl Med 12: 792-799

14. Uematsu H, Yamada H, Sadato N et al. (1998) Assessment of hepatic portal perfusion using $\mathrm{T} 2 *$ measurement of $\mathrm{Gd}$ DTPA. J Magn Reson Imaging 8: 650-654

15. Rubin JM, Adler RS, Fowlkes JB et al. (1995) Fractional moving blood volume: estimation with power Doppler US. Radiology 197: 183-190

16. Rubin JM, Bude RO, Fowlkes JB, Spratt RS, Carson PL, Adler RS (1997) Normalizing fractional moving blood volume estimates with power Doppler US: defining a stable intravascular point with the cumulative power distribution function. Radiology 205: 757-765

17. Meier P, Zierler KL (1954) On the theory of the indicator-dilution method for measurement of blood flow and volume. J Appl Physiol 6: 731-744

18. Axel L (1980) Cerebral blood flow determination by rapid-sequence computed tomography: theoretical analysis. Radiology 137: 679-686

19. Fowlkes JB, Sirkin DW, Ivey JA, Gardner EA, Rhee RT, Rubin JM, Carson PL (1998) Transcutaneous interruption of ultrasound contrast agents for blood flow evaluation. Invest Radiol 33: 893-901

20. Blomley MJK, Albrecht T, Cosgrove DO et al. (1999) Improved imaging of liver metastases with stimulated acoustic emission in the late phase of enhancement with the US contrast agent SHU 508A: early experience. Radiology 210: 409-416

21. Powers JE, Burns PN, Souquet J (1997) Imaging instrumentation for ultrasound contrast agents. In: Nanda NC, Schlief R, Goldberg BB (eds) Advances in echo imaging using contrast enhancement. Kluwer, Dordrecht, pp 139-171

22. Burns PN, Simpson DH (1998) Pulse inversion Doppler: a new ultrasound technique for nonlinear imaging of microbubble contrast agents and tissue. Radiology 209(P):190

23. Atkinson P, Berry MV (1974) Random noise in ultrasonic echoes diffracted by blood. Phys A Math Nucl Gen 7: 1293-1302

24. Bamber J, Hasan P, Cook-Martin G, Bush N (1988) Parametric imaging of tissue shear and flow using B-scan decorrelation rate. J Ultrasound Med 7:S135

25. Chen J, Fowlkes JB, Carson PL, Rubin JM (1996) Determination of scan-plane motion using speckle decorrelation: theoretical considerations and initial test. Int J Imaging Syst Technol 8: $38-44$ 